UTILIZING MULTIMODAL ANALGESIA

\title{
Utilizing multimodal analgesia to evaluate postoperative analgesic requirements in kidney transplant recipients
}

By

Michele A. Muir, PharmD Candidate ${ }^{1}$

Co-authors: Kristen R. Szempruch, PharmD, BCPS ${ }^{2}$, Robert Dupuis, PharmD, FCCP 1 Alexander H. Toledo, $\mathrm{MD}^{3}$, Robert S. Isaak, DO, FASA ${ }^{4}$, Harendra Arora, MD, MBA, FASA ${ }^{4}$, Ravindra Prasad, $\mathrm{MD}^{4}$, Pablo Serrano Rodriguez, MD, FACS ${ }^{3}$

${ }^{1}$ Department of Pharmacotherapy and Experimental Therapeutics, UNC Eshelman School of Pharmacy, Chapel Hill, NC

${ }^{2}$ Department of Pharmacy, UNC Carolina Medical Center, Chapel Hill, NC

${ }^{3}$ Department of Surgery, Abdominal Transplant, UNC Medical Center, Chapel Hill, NC

${ }^{4}$ Department of Anesthesiology, UNC Medical Center, Chapel Hill, NC

\author{
Honors Thesis \\ UNC Eshelman School of Pharmacy \\ University of North Carolina at Chapel Hill
}

January 25,2021

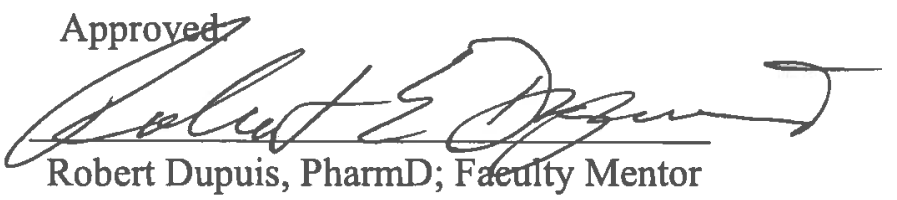


UTILIZING MULTIMODAL ANALGESIA

\begin{abstract}
The use of non-opioid analgesics following surgery has proven beneficial in managing pain and decreasing adverse outcomes following surgery. Data assessing outcomes related to opioid use is limited in kidney transplant recipients (KTRs). We evaluated the effectiveness of implementing a reduced to no opioid use protocol in KTRs. This retrospective cohort study included adult KTRs between January 2017 and July 2019 with a multimodal analgesic protocol (MAP), focused on limiting opioids, implemented in August 2018. We compared analgesic requirements in morphine milligram equivalents (MME) during transplant admissions between the MAP cohort and traditional cohort. There were 217 KTRs who met criteria. Inpatient opioid use was significantly reduced in the MAP cohort $(16.5 \pm 19.2 \mathrm{MME} /$ day vs $24.7 \pm 19.7 \mathrm{MME} / \mathrm{day} ; \mathrm{p}$ $<0.05)$ with no significant difference in pain scores. No use of opioids within six months of discharge was significantly increased in the MAP cohort $(50 \%$ vs $7 \%$; $p<0.001)$ and there were no reported deaths at six months in either cohort. The use of multimodal analgesia is beneficial in KTRs to provide adequate pain control with limited to no exposure of opioids during admission or at discharge.
\end{abstract}


UTILIZING MULTIMODAL ANALGESIA

\section{Introduction}

Many common surgeries and diseases are associated with an increased risk of chronic opioid use, including patients with kidney failure who may experience pain that is multifactorial in nature or related to concomitant comorbidities. ${ }^{1-3}$ Inadequate pain control has been associated with prolonged hospital length of stay (LOS), increased readmission secondary to pain, and decreased quality of life. ${ }^{1}$ One promising strategy for reducing opioid use in the operative setting is the use of multimodal analgesia extrapolated from an enhanced recovery after surgery pathway (ERAS). The ERAS pathways utilize multimodal analgesia and focus on a set of protocols implemented before, during, and after surgery to ensure optimal outcomes. The intended outcomes include reduction or elimination of opioid use postoperatively or at discharge. Compared with traditional management, multimodal analgesia can reduce opioid use, in the immediate and long term postsurgical periods. ${ }^{4}$ To date, there is minimal data published on minimizing and reducing opioid use postoperatively in this surgical transplant population.

Existing literature has elucidated the negative effects of opioids in the transplant population. The use of pre-surgical opioid analgesics has been increasingly recognized as a predictor of postoperative complications and resource utilization in a variety of surgical populations, including general, orthopedic, and liver transplant surgeries ${ }^{5-7}$ Opioid use prior to kidney transplantation was strongly associated with inferior one-year post-transplant outcomes. High-level opioid use, defined as $>1,000$ morphine milligram equivalents (MME) per year, predicted a 46\% increased risk of death and $28 \%$ increased risk of all-cause graft failure. ${ }^{8}$ A similar retrospective study assessing chronic opioid use prior to transplant found an increased mortality risk noting that additional studies are warranted to understand the mechanisms. ${ }^{9}$ Comparably, opioid use post- 
UTILIZING MULTIMODAL ANALGESIA

transplantation showed similar trends of opioid use prior to transplant. Recipients with increased postoperative opioid requirements exhibit increased risk of death and graft failure in the first year after transplant. ${ }^{2}$ Effects of high-level opioid use in the first year after transplant, predicted twice the risk of death and $68 \%$ higher all-cause graft failure over the subsequent year, with an increased risk persisting over five years compared to no use. ${ }^{8}$ These outcomes further exemplify the need to implement opioid limiting regimens in KTRs.

\section{Multimodal Analgesia}

Multimodal analgesic agents, such as ketamine, gabapentinoids, and tramadol, have been evaluated and supported in the literature for perioperative and postoperative use in a variety of surgical patients. Ketamine is an N-methyl-d-aspartate (NMDA) receptor antagonist that has been used in the setting of chronic, cancer, neuropathic, and postsurgical pain due to the NMDA receptor blockade that results in decreased nociceptive and inflammatory pain transmission. ${ }^{10} \mathrm{~A}$ large systematic review including 130 studies with 8341 patients evaluated the efficacy and safety of perioperative intravenous ketamine in adult patients undergoing various surgical procedures. The study found perioperative intravenous ketamine reduced postoperative opioid consumption over 24 hours by $8 \mathrm{MME}$ and over 28 hours by $13 \mathrm{MME}$, when compared to placebo. Additionally, perioperative intravenous ketamine reduced pain at rest at 24 hours by $5 / 100 \mathrm{~mm}$ on visual analogue scale and at 48 hours by 5/100 $\mathrm{mm}$. Results were consistent when analyzed by pain at rest or on movement, operation type, and timing of administration, or sensitivity to study size and pain intensity. In regards to safety, ketamine bolus doses of $0.25-1$ $\mathrm{mg} / \mathrm{kg}$ and infusions $2-5 \mu \mathrm{g} / \mathrm{kg} /$ minute produced no increased risk of central nervous system side effects when compared to placebo. ${ }^{11}$ 
Although initially developed as an antiepileptic drug, gabapentin has analgesic properties through its interaction with $\alpha-2-\delta$-subunits of voltage-gated calcium channels, decreasing the release of excitatory neurotransmitters. ${ }^{12}$ Through a similar mechanism, pregabalin has also been evaluated for its reduction in perioperative opioid consumption and pain scores. Several meta analyses found that pregabalin leads to a significant reduction in pain scores and perioperative opioid consumption. ${ }^{13-15}$

Tramadol is a synthetic analogue of the opioid drug codeine; however, the analgesic potency is found to be ten times less than morphine. Tramadol is considerably safer, as it is significantly less likely to cause respiratory depression and addiction when compared to other opioid analgesics. ${ }^{16}$ It produces analgesia through various pathways such as a weak mu-opioid receptor agonist or as a serotonin and norepinephrine reuptake inhibitor for nociception. ${ }^{17,18}$ Tramadol has significant benefit due to its dual mechanism of action and minimal side effect profile. As with all medications, patient specific factors and clinical judgement should play a role in appropriate analgesic management. For the purposes of this study, tramadol was not considered an "opioid" and was a key component of the multimodal analgesia regimen.

The majority of research regarding pain management in kidney transplant patients has focused primarily on kidney transplant donors, with relatively little known about the postoperative analgesic management of KTRs. This study will address several outcomes that have not been collectively reviewed in previous studies. The aim of this study was to examine the pain management outcomes utilizing a Multimodal Analgesia Protocol (MAP) in KTRs. 


\section{Materials and Methods}

\section{Study design}

This was a single-center, retrospective cohort study conducted at our institution between January 2017 and July 2019 with the implementation of MAP (Figure 1) in August 2018. This protocol was standardized as all transplants were performed by the same group of surgeons. The traditional cohort, consisting of those transplanted prior to August 2018, was compared to the MAP cohort which was defined as those transplanted following the MAP implementation. The traditional cohort was discharged on as needed acetaminophen and oxycodone, regardless of their pain score at the time of discharge or their use of opioids prior to the transplant. The MAP cohort was discharged on acetaminophen, gabapentin, and tramadol. For the purposes of this study, tramadol was not considered an "opioid" although we acknowledge its mechanism of action as being "opioid-like" and it being considered a controlled substance. Follow-up was concluded at six months following discharge. This study was approved by the Institutional Review Board (protocol number 19-1408), which reviewed the ethical, scientific, and medical appropriateness of the study before commencement.

\section{Transplant Recipients}

Adults ( $\geq 18$ years of age) who were recipients of a kidney transplant from a living or deceased donor were considered eligible. Exclusion criteria included those who underwent dual organ transplantation.

\section{Endpoints}


UTILIZING MULTIMODAL ANALGESIA

The primary study objective was to assess the opioid use during admission defined as the preoperative through postoperative periods, quantified in MME. Secondary efficacy endpoints included recipient reported pain severity scores (scale 0-10), LOS, amount of opioid use within six months prior to and following transplantation, 30-day readmission rates and 6-month patient survival. Secondary safety endpoints included intolerance or adverse drug events due to multimodal analgesia medications.

\section{Procedures}

In the traditional cohort, recipients received fentanyl intraoperatively, while postoperatively had intermittent intravenous fentanyl available on an as needed basis. For the traditional cohort, both post-operative agent, dose, and duration were subject to surgeon discretion. Recipients were discharged on oxycodone $5 \mathrm{mg}$ orally every four to six hours as needed for moderate to severe pain in addition to acetaminophen $325 \mathrm{mg}$ orally every four to six hours as needed for mild pain or fever.

To minimize opioids, the MAP cohort recipients received preoperative, intraoperative and postoperative analgesia management as described below.

\section{Preoperative}

Multimodal analgesia of acetaminophen $1000 \mathrm{mg}$ orally and pregabalin $50 \mathrm{mg}$ orally were administered. A pain coping skills workbook was provided to all KTRs, along with transplant psychologist recommendations.

\section{Intraoperative}


UTILIZING MULTIMODAL ANALGESIA

A ketamine bolus $0.5 \mathrm{mg} / \mathrm{kg}$ was administered after induction of anesthesia, followed by a 0.25 $\mathrm{mg} / \mathrm{kg} / \mathrm{hr}$ infusion. The surgical team performed a transversus abdominis plane (TAP) block with $40 \mathrm{~mL}$ of $0.25 \%$ bupivacaine prior to abdominal closure. The goal during intraoperative pain management was to utilize multimodal analgesia to minimize intravenous opioid use. However, exceptions for chronic opioid users were considered, as deviations from MAP due to tolerance from previous chronic opioid use could occur.

\section{Postoperative}

Analgesia regimens were dependent on pain severity. Oral acetaminophen $1000 \mathrm{mg}$ three times daily was given for a minimum of five days postoperatively and then transitioned to as needed dosing. Oral gabapentin $100 \mathrm{mg}$ was initially administered daily with dose and frequency adjustments based on renal function for a duration of fourteen days. Oral tramadol 50 every six hours as needed for moderate pain or $100 \mathrm{mg}$ every six hours for severe pain could be given. The total dose and frequency of tramadol was adjusted based on recipient's renal function. If tramadol was contraindicated, oral oxycodone $5 \mathrm{mg}$ every six hours as needed for moderate pain and $10 \mathrm{mg}$ every six hours as needed for severe pain were considered. Intravenous (IV) fentanyl $50 \mathrm{mcg}$ every six hours as needed for two doses was administered for severe pain with reassessment of pain when requiring more than two doses in a 24-hour period. If pain was not well controlled, fentanyl could be adjusted to 50-100 mcg IV every four hours as needed. Additionally, recipients who were on chronic opioids preoperatively were continued on their home pain medication regimen.

\section{Discharge}

Pain management included tramadol $50 \mathrm{mg}$ orally every six hours as needed for moderate pain and $100 \mathrm{mg}$ orally every six hours as needed for severe pain for five days; dose and frequency 
UTILIZING MULTIMODAL ANALGESIA

were adjusted based on renal function. If tramadol was contraindicated, oxycodone orally 5-10 $\mathrm{mg}$ every six hours as needed was prescribed. Oral gabapentin $100 \mathrm{mg}$ daily for two weeks was prescribed based on renal function. Oral acetaminophen $1000 \mathrm{mg}$ three times daily for five days was provided or as long as the recipient was taking tramadol or opioids. Recipients were asked to bring unused medication to follow up clinic visits.

Data was obtained via chart review using the Epic Electronic Health Record System. Opioid use throughout admission was converted to MMEs and standardized by length of hospital stay. Opioid prescriptions within six months prior to admission and within six months following discharge were collected based on outpatient prescriptions that were recorded in the medical record. The sum of MME within those six months were categorized into MME levels (No use, Level 1: >0 to 150mg, Level 2: 151-300mg, Level 3: 301-500mg, Level 4: $>500 \mathrm{mg}){ }^{8}$ No use was defined as no opioid use at any time within the six-month time frame and tramadol was not evaluated as an opioid for this measurement. MME conversion factors were standardized using the Center for Disease Control and Prevention resource for analyzing prescription data. ${ }^{19}$ Additionally, daily recipient reported pain scores were assessed using a numeric (0-10) pain rating scale and averaged based on hospital length of stay. KTRs who did not follow MAP were included in the MAP cohort and defined as those receiving opioids beyond postoperative day one, receiving opioids prior to admission, or were discharged with an opioid prescription.

\section{Statistical Analyses}

Parametric and nonparametric data are presented as means \pm standard deviation (SD) or median with interquartile range (IQR), respectively. For parametric continuous variables, we used the 
UTILIZING MULTIMODAL ANALGESIA

unpaired $t$ test. Categorical variables were presented as frequencies and percentages. Chi-square test with Yates Correction or Fisher exact test as appropriate were used to analyze nominal and categorical data. Mood's Median test was used to compare median values. Two-tailed p-values $<0.05$ were considered statistically significant.

\section{Results}

\section{Recipient disposition and demographics}

Between January 2017 and July 2019, 217 consecutive KTRs were analyzed. There were 147 KTRs in the traditional cohort and 70 KTRs in the MAP cohort. There were no differences in recipient characteristics between the traditional and MAP cohorts (Table 1). Nor were there differences between KTRs who followed the MAP and those who did not follow the MAP protocol (Table 2).

\section{Traditional vs MAP}

Endpoints are outlined in Table 3. The overall MME during admission was significantly reduced in the MAP cohort (mean 16.5; SD 19.7 MME/day) compared with the traditional cohort (mean 24.7; SD 19.2 MME/day) ( $\mathrm{p}<0.01)$. Opioid use in deceased donor KTRs in the MAP cohort (mean 14.5; SD 18.3 MME/Day) was significantly less compared to deceased donor KTRs in the traditional cohort (mean 25.5; SD 21.6 MME/Day) $(\mathrm{p}<0.01)$. There was no significant difference in overall MME between cohorts in KTRs of living donors.

There was no significant difference in recipient reported pain scores between cohorts. Decreased length of stay was observed in recipients who followed the MAP (median 5, IQR 4-7 days) 
UTILIZING MULTIMODAL ANALGESIA

compared to the traditional cohort (median 6, IQR 5-8 days), although not statistically

significant. Opioid use within six months post discharge was significantly reduced in the MAP cohort for all but level 2 opioid use. Up to $50 \%$ of recipients in the MAP cohort did not have any outpatient opioid use within six months of discharge compared to only $7 \%$ in the traditional cohort $(\mathrm{p}<0.001)$. Of the traditional cohort, $25 \%$ had level 4 opioid use and $61 \%$ had level 3 opioid use within the timeframe, while the MAP cohort significantly decreased to $9 \%(p<0.05)$ and $21 \%(p<0.001)$, respectively. The rate of readmission was significantly decreased in the MAP cohort $(21 \%$ vs $38 \% ; \mathrm{p}<0.05)$ and time to readmission trended longer in the MAP cohort (mean 11.8, SD 10.5 days vs mean 8.5 , SD 6.9 days) but was not significant. There were no deaths within six months of discharge in either cohort.

\section{Followed MAP vs Did Not Follow MAP}

Endpoints are outlined in Table 4. KTRs who followed the MAP $(n=40)$ had significantly less opioid use throughout transplant admission (mean 7; SD 4.1 MME/day vs mean 29; SD 23.8 MME/day) $(\mathrm{p}<0.01)$ than KTRs who deviated from the protocol $(\mathrm{n}=30)$. Length of hospital stay was lower in KTRs who followed the MAP (median 5; IQR 4-7 days vs median 6.5; IQR 5-8) days) although it did not reach significance. There was no significant difference in recipient reported pain scores between these recipients. Of those who did not follow the protocol, $23 \%$ had an active opioid prescription within the six months leading up to transplant compared to $10 \%$ who followed the MAP protocol. Up to $60 \%$ of these recipients received oxycodone and $40 \%$ received intravenous opioids beyond postoperative day one. Despite not following the protocol, $30 \%$ did not receive an opioid prescription upon discharge. 
UTILIZING MULTIMODAL ANALGESIA

\section{Safety Endpoints}

There was one adverse drug event due to gabapentin in the MAP cohort that included lip, throat, and facial swelling. There were no readmissions related to opioid use in the MAP cohort; however, two recipients in the traditional cohort were readmitted for nausea, vomiting, or constipation which resulted in the oxycodone being discontinued.

\section{Discussion}

By exposing the patient to less opioids during admission and at discharge, recipients not only had comparable pain scores that demonstrated adequate pain control but had significantly less opioids during the six-month period following transplant. Similarly in a previous cohort analysis of living kidney donors, the ERAS cohort utilizing a similar multimodal analgesic approach had a $40 \%$ reduction in morphine dose equivalents compared to the traditional cohort with similar pain scores. ${ }^{20}$ These findings align with previous results showing multimodal analgesia reduced opioid use in both the immediate and long term post-surgical periods. ${ }^{4} \mathrm{~A}$ cross-sectional comparative study comparing hemodialysis patients with deceased donor KTRs found that a successful kidney transplantation did not lead to a significant reduction in pain prevalence when compared to chronic hemodialysis patients and pain relief medication are underused in kidney transplant recipients. Therefore, it is essential to manage pain with non-opioid analgesics to reduce adverse and potentially fatal adverse effects of opioid analgesics. ${ }^{21}$ By following a no opioid to limited opioid MAP protocol at discharge, the MAP cohort had significantly less opioid use within six months following discharge. There was a significant increase in those who had "No use" or Level 1 use in the MAP cohort and a significant decrease in Level 3 or higher opioid use in the six-month period. Our study supports the use of multimodal analgesia to minimize or 
UTILIZING MULTIMODAL ANALGESIA

eliminate opioid use in KTRs during transplant admission as adequate pain control and decreased outpatient opioid prescriptions can play a role in reducing the serious risks of adverse drug events due to opioids such as addiction, respiratory depression, and even death.

It should be noted that the overall decrease in MME during admission appears to be driven by deceased donor KTRs. The difference between living and deceased donor recipients is multifactorial, but we can speculate a confounding factor could have been a more structured preoperative and postoperative medication regimen. Living donor recipients have scheduled transplantation surgeries which can allot time to lower the recipient's MME or set a goal prior to the procedure. Contrasting, deceased donor recipients can wait several years before a kidney is immediately available, which makes managing preoperative opioid levels challenging. Additionally, deceased donor kidneys may require continued dialysis which can precipitate uremia and edema, contributing towards the recipient's pain. Further research is needed to elucidate this difference. However, we continue to see the beneficial effects multimodal analgesia has compared to standard opioid pain management that previously included intraoperative fentanyl with a continued opioid infusion postoperatively.

\section{MAP Deviation}

Patients were included in this study regardless of preoperative opioid use to provide insight into managing patients with multimodal analgesics that may have varying levels of opioid dependence. Of note, $23 \%$ of those who deviated from the protocol had an outpatient opioid prescription upon admission, suggesting either pre-existing chronic use or opioid tolerance. The use of opioids beyond postoperative day one, whether oral oxycodone or IV opioids, appeared to 
UTILIZING MULTIMODAL ANALGESIA

result in a continuation of opioids at discharge. Despite failing the protocol, $30 \%$ of recipients that received opioids beyond postoperative day one was not discharged with an opioid prescription.

A study exploring the metrics that influence transplant related costs found increased length of stay resulted in an increase in transplant related costs by US $\$ 3,422$ per additional day. ${ }^{22}$ KTRs who did not deviate from the protocol had a decreased length of stay by approximately one day; although not statistically significant, we believe this to be clinically and financially significant. The rate of readmissions was also significantly reduced which could suggest better postoperative pain management with multimodal analgesia, however, this is only an association as we acknowledge several other confounding factors can contribute to readmission. We cannot neglect the concerns that dialysis patients and recent kidney transplant recipients are at a greater risk of opioid-related toxicities due to altered drug protein binding, metabolism, and excretion that can lead to accumulation of these opioids and potentially toxic metabolites. ${ }^{23-26}$ Additionally, opioid use may be related to riskier behaviors such as non-adherence to medications or follow-up appointments which may contribute to adverse post-transplant events. ${ }^{27}$

\section{Multimodal Analgesia}

Multimodal analgesia methods are utilized in all phases of surgery to provide optimal pain control and accelerate recovery. The TAP block performed intraoperatively provides analgesia during surgery and for a short period following surgery and has been beneficial in managing pain for diverse surgical populations. ${ }^{28-30}$ There were no complications related to the TAP block and this is routinely performed for multiple procedures within our institution. 
UTILIZING MULTIMODAL ANALGESIA

Intravenous ketamine is frequently used intraoperatively in a variety of surgical populations since it has been shown to reduce postoperative opioid consumption and decrease pain independent of operation type or timing administration without an increased risk for central nervous system side effects. ${ }^{11}$ In the postoperative setting, fentanyl is a rapidly titratable, short acting opioid that was used on an as needed basis for patients with severe breakthrough pain that was not adequately treated with the multimodal analgesia regimen in place. Ketamine was not chosen to fulfill this role postoperatively due to the need for continuous infusion and requirement for a pain consultation. Therefore, fentanyl in the immediate postoperative setting allows for zero-use or some use up to 24 hours postoperatively.

Amongst the gabapentinoids, pregabalin was selected for preoperative pain management due to its predictable pharmacokinetic profile in comparison to gabapentin and because its oral absorption is both extensive and proportional to dose. ${ }^{13}$ On the other hand, gabapentin was selected postoperatively due to its lower cost and easier access for patients. For some patients, pregabalin may require a prior insurance authorization or be more costly in the outpatient setting.

Although tramadol is opioid-like and interacts with similar receptors as opioids, it has a safer side effect profile and lower addiction potential. ${ }^{16,17}$ In the MAP, tramadol is prescribed with a five-day supply on an as needed basis to adequately treat postoperative associated pain. Although it may be a limitation to not include MME associated with tramadol, our ultimate goal is not to be opioid free but to provide safe care to our patients while providing adequate analgesia. 
UTILIZING MULTIMODAL ANALGESIA

The medical team works closely with psychology, social work, pain clinics, and patients to guide the patient into a good MME threshold prior to transplant. Tools and resources within the MAP, such as the pain coping workbook and transplant psychology, are valuable in aiming to reduce postoperative opioid consumption.

The various modalities utilized in the MAP are not new to medical practice; however, when they are structured in a patient specific care pathway, positive results regarding pain control are achieved. By assessing the postoperative analgesic requirements following kidney transplantation, we are able to evaluate the impact of multimodal analgesia compared to the previous standard of treatment that utilized opioids freely, thus reducing negative downstream health outcomes. This study, in particular, provides valuable information on KTRs, who are eager to return to their daily routine without impairment due to pain.

We address the limited number of studies focused on opioid use in KTRs, specifically the impact of pain on transplant outcomes. Although a previous report from the United Kingdom evaluated similar outcomes of hospital LOS and associated costs, the morphine patient-controlled analgesia postoperatively does not align with the current MAP used by our institution. To our knowledge, we are one of the first to execute this study at a large academic medical center within the United States. This study helps elucidate best practices for the unique kidney transplant population and allows clinicians to effectively guide analgesic therapy for specific populations within KTRs. Implementing this protocol within our institution was not without its challenges. The biggest barrier was organizing meetings with all of the stakeholders to create the protocol and order set. Once the protocol was finalized, educating the medical team on the protocol and addressing 
UTILIZING MULTIMODAL ANALGESIA

which pain regimen to initiate following the surgical procedure was another challenge. There are still deviations when providers place orders outside of the order set and initiate a KTR on oxycodone without consulting the protocol. Another continued barrier specific to KTRs is that tramadol and gabapentin are renally dose adjusted, limiting the amount of medication that can be given for breakthrough pain when abiding by the recommended maximum dose. Patients' perceived dependence of opioids at discharge was another barrier, however this was reduced through patient education.

\section{Limitations}

Some limitations include the retrospective nature of this study and potential for confounding factors and biases. The use of historical data as our data collection is reliant on accurate documentation and charting in the medical records. Pain severity scores documented within the records are subjective measurements and can vary between recipients. The opioid prescriptions collected are limited to those sent via our electronic health record and fill histories were unable to be disclosed by the statewide prescription drug monitoring program. Therefore, external prescriptions could not be captured. Our focus on minimizing opioid use in the perioperative and postoperative periods aims to discharge KTRs without opioids or the need to prescribe them in the outpatient setting due to effective multimodal analgesia management. However, the difference in opioid use within the six months following discharge could be attributed to increased awareness surrounding opioid prescribing and alternative prescribing patterns. Our findings assessed the change in prescribing habits as providers transitioned from prescribing oxycodone at discharge to tramadol. By reducing or avoiding the use of oxycodone at discharge, we aim to decrease unnecessarily prolonged narcotic use. Our goal is to provide safe medications 
UTILIZING MULTIMODAL ANALGESIA

to our KTRs and although tramadol interacts with the same receptors as opioids, it has a safer side effect profile when compared to traditional opioids (i.e., oxycodone, hydromorphone). ${ }^{17} \mathrm{We}$ only captured readmissions to our institution, and thus could not include admissions to external facilities. Additionally, recipient comorbidities could potentially confound results, as complications associated with certain disease states can lengthen hospital stay or contribute to readmissions post-transplantation. ${ }^{31-34}$ Nonetheless, the results contribute to the limited literature on the pain management for KTRs and aid in improving the current standard of care and prevention of adverse drug reactions.

In conclusion, this retrospective analysis of patients undergoing kidney transplantation, which focuses on the use of multimodal analgesia, shows promising results in decreased opioid consumption with similar pain scores, associated decrease in length of stay, and reduced readmissions. The data gathered from this study has the potential to improve current practice during transplant admission in the kidney transplant population. Additional studies that capture outpatient opioid use are warranted to provide further insight into transplant outcomes associated with use prior to or following transplantation. Future studies may focus on methods to prevent deviation from the MAP so recipients can benefit from positive outcomes associated with reducing to eliminating opioids in the transplant process. 
UTILIZING MULTIMODAL ANALGESIA

Table 1: Baseline Characteristics for Traditional vs MAP Cohorts

\begin{tabular}{|c|c|c|c|}
\hline Characteristic $\mathbf{N}(\%)$ or Mean \pm SD & $\begin{array}{c}\text { Traditional } \\
(\mathrm{N}=147)\end{array}$ & $\begin{array}{c}\text { MAP } \\
(\mathbf{N}=70)\end{array}$ & $p$-value \\
\hline Age at Transplant, years & $49.7 \pm 13.3$ & $52.1 \pm 11.3$ & 0.21 \\
\hline Male & $82(56)$ & $40(57)$ & 0.96 \\
\hline \multicolumn{4}{|l|}{ Race } \\
\hline Caucasian & $65(44)$ & $29(41)$ & 0.81 \\
\hline African American & $72(49)$ & $32(46)$ & 0.76 \\
\hline Other & $10(7)$ & $9(13)$ & 0.22 \\
\hline \multicolumn{4}{|l|}{ Employment } \\
\hline Working & $60(41)$ & $29(41)$ & 0.93 \\
\hline \multicolumn{4}{|l|}{ Comorbidities } \\
\hline HTN & $121(82)$ & $59(84)$ & 0.87 \\
\hline DM & $51(35)$ & $22(31)$ & 0.75 \\
\hline CAD & $21(14)$ & $9(13)$ & 0.94 \\
\hline CVD & $11(7)$ & $6(9)$ & 0.99 \\
\hline Other & $27(18)$ & $11(16)$ & 0.77 \\
\hline Mean Number & $1.8 \pm 0.9$ & $1.7 \pm 1$ & 0.63 \\
\hline Dialysis & $129(88)$ & $62(\overline{89})$ & 0.86 \\
\hline \multicolumn{4}{|l|}{ Native Kidney Primary Diagnosis } \\
\hline HTN Nephrosclerosis & $33(22)$ & $16(23)$ & 0.95 \\
\hline DM & $28(19)$ & $19(27)$ & 0.24 \\
\hline IgA Nephropathy & $11(7)$ & $2(3)$ & 0.3 \\
\hline FSGS & $18(12)$ & $4(6)$ & 0.21 \\
\hline PKD & $9(6)$ & $7(10)$ & 0.46 \\
\hline Other & $48(33)$ & $22(31)$ & 0.98 \\
\hline \multicolumn{4}{|l|}{ Donor Type } \\
\hline Deceased & $101(69)$ & $45(64)$ & 0.62 \\
\hline \multicolumn{4}{|l|}{$\begin{array}{l}\text { Pre-Admission Opioids within } 6 \\
\text { Months, MME }\end{array}$} \\
\hline No Use & $137(93)$ & $59(84)$ & 0.07 \\
\hline Level 1: $>0$ to $150 \mathrm{mg}$ & $2(1)$ & $5(7)$ & 0.07 \\
\hline Level 2: 151-300mg & $1(1)$ & $2(3)$ & 0.51 \\
\hline Level 3: 301-500mg & $4(3)$ & $1(2)$ & 0.91 \\
\hline Level 4: >500mg & $3(2)$ & $3(4)$ & 0.62 \\
\hline
\end{tabular}


UTILIZING MULTIMODAL ANALGESIA

Table 2: Baseline Characteristics for Followed MAP vs Did Not Follow MAP

\begin{tabular}{|c|c|c|c|}
\hline Characteristic $\mathbf{N}(\%)$ or Mean \pm SD & $\begin{array}{l}\text { Followed MAP } \\
\qquad(n=40)\end{array}$ & $\begin{array}{l}\text { Did Not Follow } \\
\text { MAP }(\mathbf{n}=\mathbf{3 0})\end{array}$ & $p$-value \\
\hline Age at transplant, years & $53.2 \pm 10.7$ & $51.9 \pm 11.4$ & 0.63 \\
\hline Male & $24(60)$ & $16 \overline{(53)}$ & 0.75 \\
\hline \multicolumn{4}{|l|}{ Race } \\
\hline Caucasian & $13(33)$ & $16(53)$ & 0.13 \\
\hline African American & $20(50)$ & $12(40)$ & 0.56 \\
\hline Other & $7(18)$ & $2(7)$ & 0.33 \\
\hline \multicolumn{4}{|l|}{ Employment } \\
\hline Working & $17(43)$ & $12(40)$ & 0.83 \\
\hline \multicolumn{4}{|l|}{ Insurance } \\
\hline Medicare & $20(50)$ & $9(30)$ & 0.15 \\
\hline Medicaid & $2(5)$ & $2(7)$ & 0.77 \\
\hline Private & $9(23)$ & $9(30)$ & 0.66 \\
\hline Other & $9(23)$ & $10(33)$ & 0.46 \\
\hline \multicolumn{4}{|l|}{ Comorbidities } \\
\hline HTN & $36(90)$ & $23(77)$ & 0.24 \\
\hline $\mathrm{DM}$ & $14(35)$ & $8(27)$ & 0.63 \\
\hline CAD & $2(5)$ & $7(23)$ & 0.06 \\
\hline CVD & $3(8)$ & $3(10)$ & 0.71 \\
\hline Other & $3(8)$ & $8(27)$ & 0.06 \\
\hline Mean Number & $1.6 \pm 0.7$ & $2 \pm 1.3$ & 0.1 \\
\hline Dialysis & $36(90)$ & $26(87)$ & 0.96 \\
\hline \multicolumn{4}{|l|}{ Native Kidney Primary Diagnosis } \\
\hline HTN Nephrosclerosis & $9(23)$ & $7(23)$ & 0.93 \\
\hline $\mathrm{DM}$ & $12(30)$ & $7(23)$ & 0.73 \\
\hline IgA Nephropathy & $1(3)$ & $1(3)$ & 0.84 \\
\hline FSGS & $2(5)$ & $2(7)$ & 0.77 \\
\hline PKD & $4(5)$ & $3(10)$ & 1.0 \\
\hline Other & $12(30)$ & $10(33)$ & 0.97 \\
\hline \multicolumn{4}{|l|}{ Donor Type } \\
\hline Deceased & $27(68)$ & $18(60)$ & 0.69 \\
\hline \multicolumn{4}{|l|}{$\begin{array}{l}\text { Pre-Admission Opioids within } 6 \\
\text { Months, MME }\end{array}$} \\
\hline No Use & $36(90)$ & $23(77)$ & 0.24 \\
\hline Level 1: $>0$ to $150 \mathrm{mg}$ & $2(5)$ & $3(10)$ & 0.74 \\
\hline Level 2: 151-300mg & $1(2.5)$ & $1(3)$ & 0.84 \\
\hline Level 3: 301-500mg & $0(0)$ & $1(3)$ & 0.88 \\
\hline Level 4: >500mg & $1(2.5)$ & $2(7)$ & 0.80 \\
\hline
\end{tabular}

CAD, coronary artery disease; CVD, cerebrovascular disease; DM, diabetes mellitus; FSGS, focal segmental glomerulosclerosis; HTN, hypertension; IgA, immunoglobulin A; PKD, polycystic kidney disease 
UTILIZING MULTIMODAL ANALGESIA

Table 3: Endpoints for Traditional vs MAP

\begin{tabular}{|c|c|c|c|}
\hline $\begin{array}{c}\text { N(\%), Mean } \pm \text { SD, Median } \\
(\text { IQR })\end{array}$ & $\begin{array}{c}\text { Traditional } \\
(\mathrm{n}=147)\end{array}$ & $\begin{array}{c}\text { MAP } \\
(n=70)\end{array}$ & $p$-value \\
\hline MME/Day & $24.7 \pm 19.7$ & $16.5 \pm 19.2$ & $<0.01$ \\
\hline Living & $22.9 \pm 15$ & $20 \pm 20.6$ & 0.51 \\
\hline Deceased & $25.5 \pm 21.6$ & $14.5 \pm 18.3$ & $<0.01$ \\
\hline LOS, Days & $6(5-8)$ & $5.5(5-7)$ & 0.55 \\
\hline Living & $5(4-7)$ & $5(4-6)$ & 0.96 \\
\hline Deceased & $6(5-8)$ & $6(5-7)$ & 0.23 \\
\hline Pain Score & $1.7 \pm 1.4$ & $2 \pm 1.4$ & 0.2 \\
\hline \multicolumn{4}{|l|}{$\begin{array}{l}\text { Post-Discharge Opioid Use } \\
\text { within } 6 \text { Months, MME }\end{array}$} \\
\hline No Use & $10(7)$ & $35(50)$ & $<0.001$ \\
\hline Level 1: >0 To $150 \mathrm{mg}$ & $1(0.6)$ & $9(13)$ & $<\mathbf{0 . 0 0 1}$ \\
\hline Level 2: 151-300mg & $10(7)$ & $5(7)$ & 0.93 \\
\hline Level 3: 301-500mg & $89(61)$ & $15(21)$ & $<0.001$ \\
\hline Level 4: >500mg & $37(25)$ & $6(9)$ & $<0.01$ \\
\hline Readmissions, days & $56(38)$ & $15(21)$ & $<0.05$ \\
\hline Time to Readmission & $8.5 \pm 6.9$ & $11.8 \pm 10.5$ & 0.12 \\
\hline
\end{tabular}

IQR, interquartile range; LOS, length of stay; MME, milligram morphine equivalent 
UTILIZING MULTIMODAL ANALGESIA

Table 4: Endpoints for Followed MAP vs Did Not Follow MAP

\begin{tabular}{|c|c|c|c|}
\hline $\begin{array}{l}\text { N(\%), Mean } \pm \text { SD } \\
\text { Median }(\text { IQR })\end{array}$ & $\begin{array}{l}\text { Followed MAP } \\
\qquad(n=40)\end{array}$ & $\begin{array}{c}\text { Did Not Follow MAP } \\
(n=30)\end{array}$ & $p$-value \\
\hline MME/Day & $7 \pm 4.1$ & $29 \pm 23.8$ & $<\mathbf{0 . 0 0 1}$ \\
\hline Living & $8 . \overline{1}+4$ & $33.1+23.3$ & $<0.001$ \\
\hline Deceased & $6.5 \pm 4.1$ & $26.3 \pm 24.4$ & $<\mathbf{0 . 0 0 1}$ \\
\hline LOS, days & $5(\overline{4}-7)$ & $6.5 \overline{(5-8)}$ & 0.23 \\
\hline Living & $4(4-5)$ & $5(4-10.5)$ & 0.41 \\
\hline Deceased & $6(4-7)$ & $7(5.3-7.8)$ & 0.14 \\
\hline Pain Score Average & $1.8 \pm 1.2$ & $2.3 \pm 1.6$ & 0.14 \\
\hline \multicolumn{4}{|l|}{$\begin{array}{l}\text { Post-Discharge Opioid Use } \\
\text { within } 6 \text { Months, MME }\end{array}$} \\
\hline No use & $28(70)$ & $7(23)$ & $<\mathbf{0 . 0 0 1}$ \\
\hline Level 1: $>0$ to $150 \mathrm{mg}$ & $8(20)$ & $1(3)$ & 0.09 \\
\hline Level 2: 151-300mg & $2(5)$ & $3(10)$ & 0.74 \\
\hline Level 3: 301-500mg & $2(5)$ & $13(43)$ & $<0.001$ \\
\hline Level 4: $>500 \mathrm{mg}$ & $0(0)$ & $6(20)$ & $<\mathbf{0 . 0 5}$ \\
\hline Readmissions, days & $8(20)$ & $7(23)$ & 0.97 \\
\hline Time to Readmission & $11.5 \pm 11$ & $12.1 \pm 22$ & 0.95 \\
\hline
\end{tabular}


UTILIZING MULTIMODAL ANALGESIA

Figure 1: MAP of Analgesia Methods

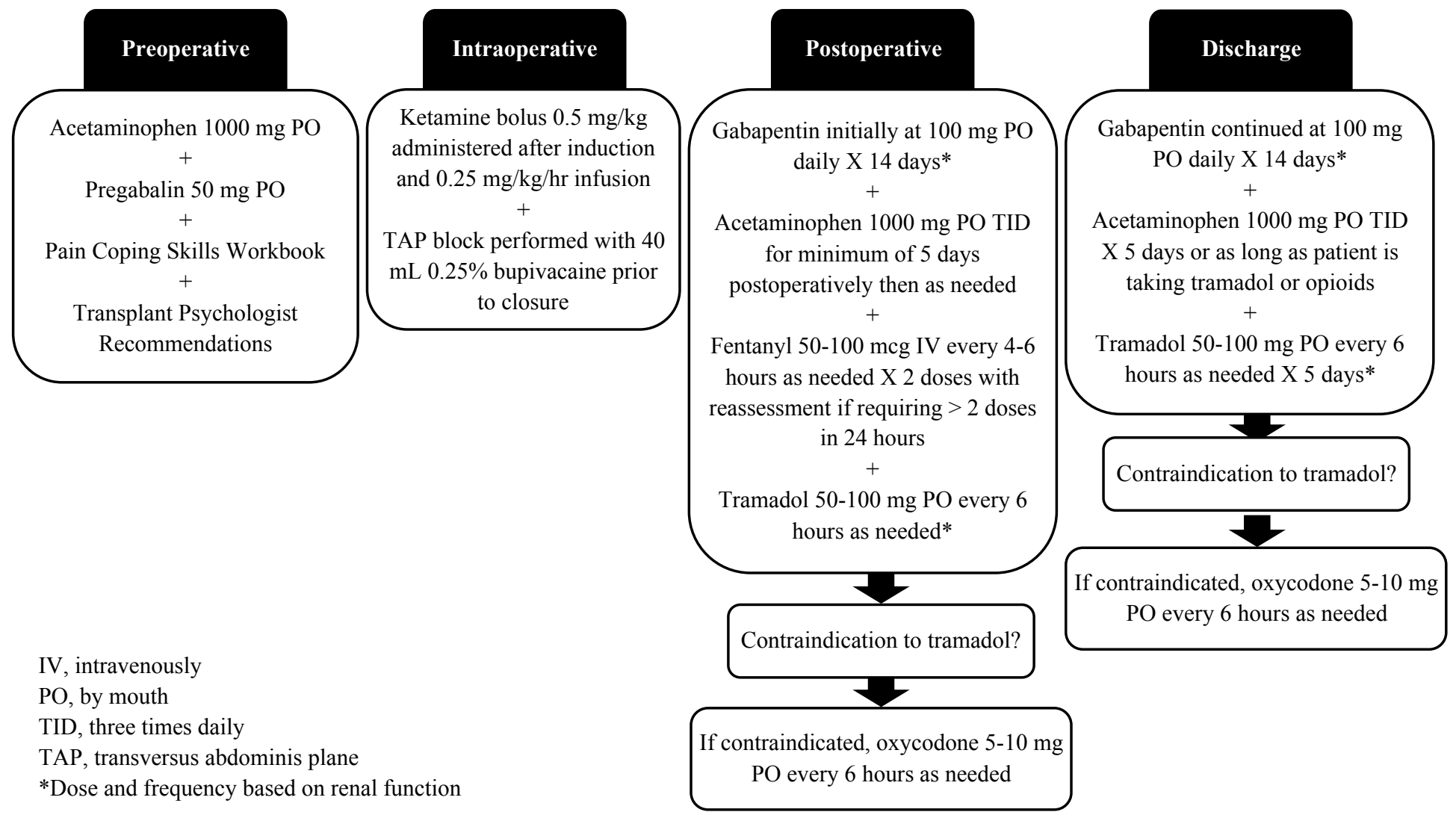

Figure 1: The MAP involved multimodal analgesia medication implementation in the

preoperative, intraoperative, postoperative, and discharge segments of care in accordance with decreasing to eliminating opioid use throughout transplant admission. 
UTILIZING MULTIMODAL ANALGESIA

\section{Acknowledgements}

The authors gratefully acknowledge Al Wagner for performing and providing data pulls for our study. Additionally, the authors acknowledge Dr. Julie Dumond, PharmD and Dr. Aaron Devanathan, PharmD, who provided guidance on manuscript revisions and statistical analyses.

\section{Authors and Contributions}

Michele A Muir ${ }^{1}$ : Participated in research design, writing of the paper, and data analysis.

Kristen R Szempruch, PharmD, BCPS 2 : Participated in research design, performance of the research, writing of the paper, and data analysis.

Robert Dupuis, PharmD, FCCP ${ }^{1}$ : Participated in research design, writing of the paper, and data analysis.

Alexander $\mathrm{H}$ Toledo, $\mathrm{MD}^{3}$ : Participated in performance of the research.

Robert S Isaak, DO, FASA ${ }^{4}$ : Participated in performance of the research.

Harendra Arora, MD, FASA ${ }^{4}$ : Participated in performance of the research.

Ravindra Prasad, $\mathrm{MD}^{4}$ : Participated in performance of the research.

Pablo Serrano Rodriguez, MD, FACS ${ }^{3}$ : Participated in performance of the research.

\section{Conflicts of Interest}

The authors declare no conflicts of interest.

\section{Funding Support}

The authors did not receive any funding for the accomplishment of this study. 


\section{References}

1. American Society of Anesthesiologists Task Force on Acute Pain Management. Practice guidelines for acute pain management in the perioperative setting: an updated report by the American Society of Anesthesiologists Task Force on Acute Pain Management. Anesthesiology. 2012;116(2):248-273.

2. Lentine KL, Lam NN, Xiao H, et al. Associations of pre-transplant prescription narcotic use with clinical complications after kidney transplantation. Am J Nephrol. 2015;41(2):165-176.

3. Brummett CM, Waljee JF, Goesling J, et al. New Persistent Opioid Use After Minor and Major Surgical Procedures in US Adults. JAMA Surg. 2017;152(6):e170504.

4. Halawa A, Rowe S, Roberts F, et al. A Better Journey for Patients, a Better Deal for the NHS: The Successful Implementation of an Enhanced Recovery Program After Renal Transplant Surgery. Exp Clin Transplant. 2018;16(2):127-132.

5. Menendez ME, Ring D, Bateman BT. Preoperative Opioid Misuse is Associated With Increased Morbidity and Mortality After Elective Orthopaedic Surgery. Clin Orthop Relat Res. 2015;473(7):2402-2412.

6. Cron DC, Englesbe MJ, Bolton CJ, et al. Preoperative Opioid Use is Independently Associated With Increased Costs and Worse Outcomes After Major Abdominal Surgery. Ann Surg. 2017;265(4):695-701.

7. Randall HB, Alhamad T, Schnitzler MA, et al. Survival implications of opioid use before and after liver transplantation. Liver Transpl. 2017;23(3):305-314.

8. Lentine KL, Lam NN, Naik AS, et al. Prescription opioid use before and after kidney transplant: Implications for posttransplant outcomes. Am J Transplant. 2018;18(12):29872999.

9. Barrantes F, Luan FL, Kommareddi M, et al. A history of chronic opioid usage prior to kidney transplantation may be associated with increased mortality risk. Kidney Int. 2013;84(2):390-396.

10. Jouguelet-Lacoste J, La Colla L, Schilling D, Chelly JE. The use of intravenous infusion or single dose of low-dose ketamine for postoperative analgesia: a review of the current literature. Pain Med. 2015;16(2):383-403.

11. Brinck EC, Tiippana E, Heesen M, et al. Perioperative intravenous ketamine for acute postoperative pain in adults. Cochrane Database Syst Rev. 2018;12:CD012033.

12. Rose MA, Kam PCA. Gabapentin: pharmacology and its use in pain management. Anaesthesia. 2002;57(5):451-462.

13. Lam DMH, Choi S-W, Wong SSC, Irwin MG, Cheung C-W. Efficacy of Pregabalin in Acute Postoperative Pain Under Different Surgical Categories: A Meta-Analysis. Medicine . 
UTILIZING MULTIMODAL ANALGESIA

2015;94(46):e1944.

14. Mishriky BM, Waldron NH, Habib AS. Impact of pregabalin on acute and persistent postoperative pain: a systematic review and meta-analysis. Br J Anaesth. 2015;114(1):1031.

15. Eipe N, Penning J, Yazdi F, et al. Perioperative use of pregabalin for acute pain - a systematic review and meta-analysis. Pain. 2015;156(7):1284.

16. Preston KL, Jasinski DR, Testa M. Abuse potential and pharmacological comparison of tramadol and morphine. Drug Alcohol Depend. 1991;27(1):7-17.

17. Scott LJ, Perry CM. Tramadol: a review of its use in perioperative pain. Drugs. 2000;60(1):139-176.

18. Ide S, Minami M, Ishihara K, Uhl GR, Sora I, Ikeda K. Mu opioid receptor-dependent and independent components in effects of tramadol. Neuropharmacology. 2006;51(3):651-658.

19. Data Resources | Drug Overdose | CDC Injury Center.

https://www.cdc.gov/drugoverdose/resources/data.html. Published October 4, 2019. Accessed March 26, 2020.

20. Campsen J, Call T, Allen CM, et al. Prospective, double-blind, randomized clinical trial comparing an ERAS pathway with ketorolac and pregabalin versus standard of care plus placebo during live donor nephrectomy for kidney transplant. Am J Transplant. 2019;19(6):1777-1781.

21. Masajtis-Zagajewska A, Pietrasik P, Krawczyk J, et al. Similar prevalence but different characteristics of pain in kidney transplant recipients and chronic hemodialysis patients. Clin Transplant. 2011;25(2):E144-E151.

22. Serrano OK, Vock DM, Chinnakotla S, et al. The Relationships Between Cold Ischemia Time, Kidney Transplant Length of Stay, and Transplant-related Costs. Transplantation. 2019;103(2):401-411.

23. Williams M, Milner QJW. Postoperative analgesia following renal transplantation - current practice in the UK. Anaesthesia. 2003;58(7):712-713.

24. Stein $\mathrm{C}$, Schäfer M, Machelska $\mathrm{H}$. Why is morphine not the ultimate analgesic and what can be done to improve it? J Pain. 2000;1(3 Suppl):51-56.

25. Carr AC, Stone PA, Serpell MG, Others. Patient-controlled morphine analgesia (PCA morphine) in cadaveric renal transplant recipients: does morphine-6-glucuronide accumulate? Br J Anaesth. 1998;81:630P.

26. Dean M. Opioids in renal failure and dialysis patients. J Pain Symptom Manage. 2004;28(5):497-504. 


\section{UTILIZING MULTIMODAL ANALGESIA}

27. Campbell CI, Kline-Simon AH, Von Korff M, Saunders KW, Weisner C. Alcohol and Drug Use and Aberrant Drug-Related Behavior Among Patients on Chronic Opioid Therapy.

Subst Use Misuse. 2017;52(10):1283-1291.

28. Carney J, McDonnell JG, Ochana A, Bhinder R, Laffey JG. The transversus abdominis plane block provides effective postoperative analgesia in patients undergoing total abdominal hysterectomy. Anesth Analg. 2008;107(6):2056-2060.

29. O'Donnell BD, McDonnell JG, McShane AJ. The transversus abdominis plane (TAP) block in open retropubic prostatectomy. Reg Anesth Pain Med. 2006;31(1):91.

30. Mukhtar K, Singh S. Transversus abdominis plane block for laparoscopic surgery. $\mathrm{Br} J$ Anaesth. 2009;102(1):143-144.

31. Woo YM, McLean D, Kavanagh D, et al. The influence of pre-operative electrocardiographic abnormalities and cardiovascular risk factors on patient and graft survival following renal transplantation. J Nephrol. 2002;15(4):380-386.

32. Gill JS, Abichandani R, Kausz AT, Pereira BJG. Mortality after kidney transplant failure: the impact of non-immunologic factors. Kidney Int. 2002;62(5):1875-1883.

33. Kasiske BL, Cangro CB, Hariharan S, et al. The evaluation of renal transplantation candidates: clinical practice guidelines. Am J Transplant. 2001;1 Suppl 2:3-95.

34. Wu C, Evans I, Joseph R, et al. Comorbid conditions in kidney transplantation: association with graft and patient survival. J Am Soc Nephrol. 2005;16(11):3437-3444. 